American Journal of Environmental Sciences 4 (3): 189-197, 2008

ISSN 1553-345X

(C) 2008 Science Publications

\title{
Effect of Earthquakes on Some Kind of Buildings
}

\author{
J. Alberto Escobar S., J. Antonio Mendoza, Roberto Gómez M. \\ Instituto de Ingeniería, Universidad Nacional Autónoma de México, Cd. Universitaria, \\ 04510 D.F., México
}

\begin{abstract}
A new method that simplifies the current static seismic torsion design procedure for buildings is proposed. Distribution of the shear forces among the storey-resistant elements designed by torsion and of the factors which affect the design eccentricity, specially accidental eccentricity, are studied. It is demonstrated that the effect of accidental eccentricity is always additive for the structural elements. There is given an in which the proposed procedure is applied; the results obtained agree with those calculated obtained with a traditional procedure of design.
\end{abstract}

Keywords: Seismic torsion, simplified analysis, code analysis, accidental eccentricity, simplified method

\section{INTRODUCTION}

The current philosophy of seismic design is based on the assumptions that structures subjected to severe earthquakes must be able to resist significant inelastic deformations without detriment to their load capacity. For asymmetric buildings these inelastic deformations arise fundamentally from the translation and rotation of their storeys. From this point of view, their behaviour should be such that besides supporting the lateral displacements they have to resist other displacements caused by torsion, all of which under the admissible storey displacement limits.

Considering the effects mentioned, the current regulations allow the analysis of building structures by taking into account only the translation of the storeys. Seismic torsion is included by distributing the shear force, due to this effect, among the resistant elements. This shear force is computed by considering a torsion moment in each storey, obtained from the product of the storey shear force and a design eccentricity expressed in terms of the accidental and structural eccentricities.

Like other torsion design regulations, the current México Federal District Code, MFDC, allows one to carry out a static analysis of building structures considering the seismic forces applied at the centre of mass of each storey; taken into account are also the corresponding torsion moments that are distributed among the resistant elements; however, forces obtained from this type of analysis are different from those computed from a three-dimensional dynamic analysis. This dynamic effect can produce an amplification of the torsional moments in structures with elastic behaviour mainly when the value of the uncoupled frequency ratio is near to one. To take into account this effect, a static eccentricity amplification factor is used.

On the other hand, the torsional moments in real buildings differ from those obtained from a dynamic analysis caused by not explicitly considered factors ${ }^{[1]}$. They can, in part, be attributed to the torsion induced by the rotational component of ground movements and to the difference in the arrival time of seismic waves at the supports of the structures, and, in part, to the difference between the values of real and calculated structural properties $^{[2-4]}$. Under these circumstances, because of inherent uncertainties in the structural properties even the nominally symmetrical structures can be affected by torsion $^{[5-9]}$. Nowadays it is practically impossible to estimate this effect denominated accidental torsion with precision. The usual way to consider this in the seismic design of structures is by including an additional torsional moment, computed in such a way that the storey shear force is displaced from its original position. This displacement of the shear force is called accidental eccentricity, which is commonly expressed as a percentage of the maximum dimension of the plan structure perpendicular to the direction action of the earthquake.

In this paper, whose aim is to simplify static seismic torsion design, the factors that affect design eccentricity are analysed, particularly accidental eccentricity. It is demonstrated that the forces caused by this effect are always additive for the resistant elements. Based on this finding, a procedure that simplifies the

Corresponding Author: J. Alberto Escobar S Instituto de Ingeniería, Universidad Nacional Autónoma de México, Cd. Universitaria 04510 D.F., México Tel. +52.55.56.23.36.12. Fax +52.55.56.16.29.22, 
static seismic torsion design of building structures is proposed and evaluated.

Torsional shear distribution: In accordance with the static seismic torsion design method, the design force, $V_{i}$, for the $i$-th resistant element in a storey must include a direct shear, $V_{d i}$, and a torsion shear, $V_{t i}$, i.e.:

$V_{i}=V_{d i} \pm V_{t i}$

In

Fig. 1 this effect is presented schematically for a simple structural system.

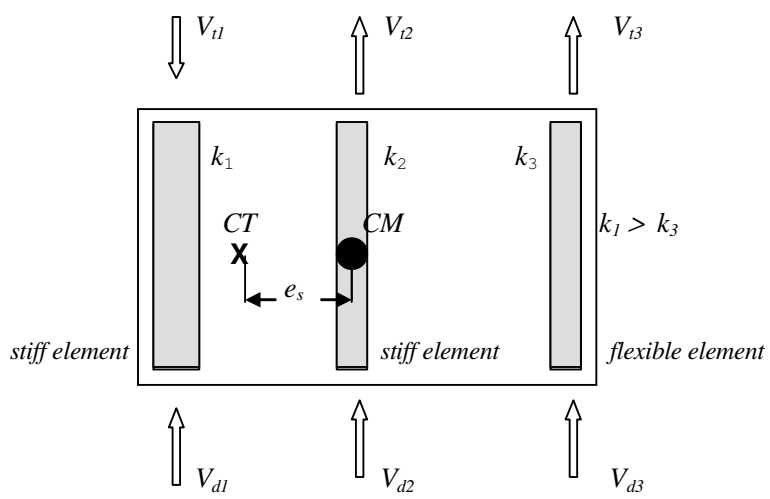

Fig. 1: Distribution of direct, $V d_{i}$, and torsion shear, $V t_{\mathrm{i}}$, among the resistant elements

Direct shear, proportional to the lateral stiffness, $k_{i}$, of each element, is calculated from the storey shear, $V_{j}$, for each one of the orthogonal axes of the structure:

$V_{d i}=V_{j} \frac{k_{i}}{\sum k_{i}}$

Torsion shear is computed from the torsional moment originating from the product of the storey shear force and a design eccentricity $e_{d}$ which can adopt two values, $e_{d l}$ and $e_{d 2}$. Most seismic-design regulations provide design eccentricity expressions with the following format:

$e_{d l}=\alpha e_{s}+\beta b$

$e_{d 2}=\delta e_{s}-\beta b$

where $\alpha$ and $\delta$ are dynamic amplification factors, $e_{s}$, is the structural or static eccentricity, defined as the distance between the centre of torsion, $C T$, and the centre of masses, $C M ; \beta$ is the accidental eccentricity factor, and $\mathrm{b}$ is the in-plan maximum dimension of the structure perpendicular to the seismic excitation direction. In accordance with the MFDC- $95^{[10]}, \alpha=1.5$, $\delta=1.0$ and $\beta=0.1$. From these equations, design eccentricity will be the one that produces the most unfavourable effects in each of the resistant elements.

Torsion design shear forces of the storey, $V t_{i}$, are obtained from the distribution of the torsional moment, taking into account the contribution of each structural element to the storey torsional stiffness, $K_{\theta}$, i.e.:

$V_{t i}=\frac{k_{i} x_{i}}{K_{\theta}} V_{j} e d i$

where $K_{\theta}=\sum x_{i}^{2} k_{i}+\sum y_{i}^{2} k_{i} ; x_{i}, y_{i}$ are the coordinates of the $i$-th structural element with respect to the torsion centre.

By incorporating the previous effects, it is possible to analyse building structures taking into account only the translation of the storeys.

Substituting equations (2) and (4) into equation (1) gives:

$V_{i}=\frac{k_{i}}{\Sigma k_{i}} V_{j} \pm \frac{k_{i} x_{i}}{K_{\theta}} V_{j} e_{d i}$

On the other hand, based on the torsional stiffness of each storey, Tso and Wong ${ }^{[1]}$ proposed a structural parameter, denominated normalized radius of gyration, $\rho$, which is quite helpful when studying the behaviour of asymmetric structures. This parameter allows one to establish minimum values of the torsional stiffness of a structure in order to maintain its storey drifts within certain acceptable limits.

When dealing with the response of structures in the non-linear range, $\rho$ can be used to control the excessive ductility demands of the resistant elements ${ }^{[11]}$.

For each of the two orthogonal axes of a structural system, the normalized radius of gyration is defined as:

$\rho=\frac{1}{b} \sqrt{\frac{K_{\theta}}{\sum k_{i}}}$

where $k_{i}$ is the stiffness of the elements in each of the orthogonal axes of the structural system. From this equation:

$$
K_{\theta}=\Sigma k_{i} \rho^{2} b^{2}
$$

Thus, from equations (5) and (7):

$$
V_{i}=V_{d i}\left(1 \pm \frac{e_{d i}}{\rho^{2} b^{2}} x_{i}\right)
$$

Substituting equations (3) in this expression, for the resistant elements located on the same side of $C M$ with respect to $C T$ (

Fig. 1), denominated "flexible

elements", it is obtained that the total design shear is given by the most unfavourable effect calculated with the following equations: 
$V_{i}=V_{d i}\left(1+\frac{\beta}{\rho^{2}} \zeta_{i}+\frac{\alpha e}{\rho^{2}} \zeta_{i}\right)$

and

$$
V_{i}=V_{d i}\left(1-\frac{\beta}{\rho^{2}} \zeta_{i}+\frac{\delta e}{\rho^{2}} \zeta_{i}\right)
$$

where $\zeta_{i}=x_{i} / b$ and $e=e_{s} / b$ are the normalized position of the $i$-th structural element and the normalized structural eccentricity, respectively. Joining equation (9) and (10), and because of the values that the parameters involved in these equations can adopt:

$$
\left.\beta b+\alpha e_{s}\right\rangle-\beta b+\delta e_{s}
$$

Therefore equation (9) will govern the design of the flexible elements.

On the other hand, the strength of the resistant elements located on the same side of the $C T$ with respect to the $C M$ (

Fig. 1), denominated "stiff elements", will be obtained by computing the most unfavourable effect with the following expressions:

$$
V_{i}=V_{d i}\left(1-\frac{\beta}{\rho^{2}} \zeta_{i}-\frac{\alpha e}{\rho^{2}} \zeta_{i}\right)
$$

And

$$
V_{i}=V_{d i}\left(1+\frac{\beta}{\rho^{2}} \zeta_{i}-\frac{\delta e}{\rho^{2}} \zeta_{i}\right)
$$

Again, joining these two equations and proceeding as above:

$$
-\beta b-\alpha e_{s}<\beta b-\delta e_{S}
$$

In this case, equation (13) will govern the design of the stiff elements; for certain values of the parameters involved in these equations, however, it is possible that equation (12) could also govern the design of this type of elements; a serious inconvenience, which will be discussed later on, appear though.

Torsional shear due to accidental eccentricity: In the MFDC, accidental eccentricity is equal to 10 percent of the maximum dimension of the structure plan perpendicular to the direction of the earthquake; thus $\beta=0.1$. The accidental torsion effect is included with a torsional moment obtained by assuming that the storey shear force moves $\pm 0.1 b$ from its original location. In this way, it is necessary to carry out a structural analysis of the building for each position of the $C M$ displaced by this quantity from its nominal position. In the case of three-dimensional structural models this procedure can lead to an excessive number of analyses. From equations (9) and (13) it can be seen that the accidental eccentricity effect is always additive, which implies that the torsion due to accidental eccentricity is transformed into an increment of the total lateral strength of the structure. This observation coincides with that previously made ${ }^{[4,11,12]}$. Thus, the effect of the accidental eccentricity for the $i$-th structural element can be represented by a Factor of Accidental Eccentricity, $F A E$, given as:

$$
F A E_{i}=\frac{\beta}{\rho^{2}} \zeta_{i}
$$

In Fig. 2 , the behaviour of this factor with respect to $\rho^{2}$ for $\beta=0.1$ and for several $\zeta_{i}$ values is presented. It can be observed that the maximum and minimum values of $F A E i$ correspond to $\zeta_{i}=1$, (which implies $\left.x_{i}=b\right)$ and for $\zeta_{i}=0.2$, respectively.

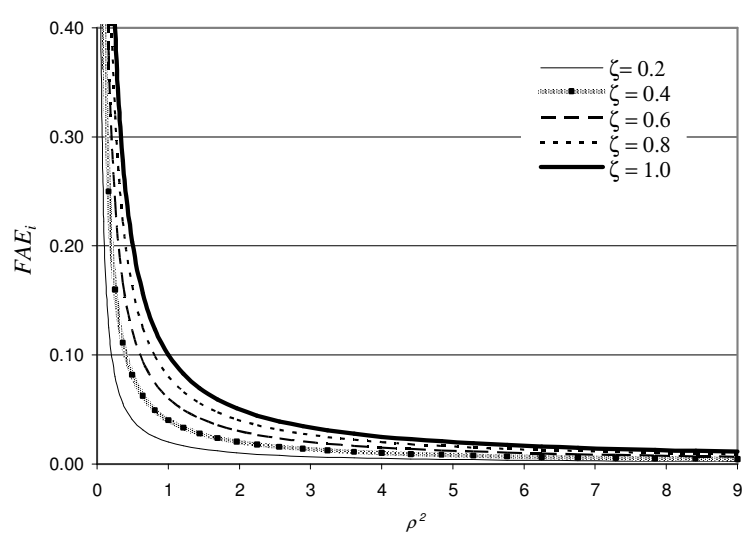

Fig. 2: Factor of Accidental Eccentricity, $F A E_{I}$, for different values of $\zeta_{i}$

Torsional Shear Due To Static Eccentricity: From equations (9) and (13), the effect of the torsion due to static eccentricity in the design of the resistant elements can be computed by using a Factor of Structural Eccentricity, FSE. Thus, from equation (9) the effect of the static eccentricity for the flexible elements is given as

$$
\operatorname{FSEf}_{i}=\frac{\alpha e}{\rho^{2}} \zeta_{i}
$$

and from equation (13), FSE for the stiff elements results in

$$
\text { FSEf }_{i}=\frac{\delta e}{\rho^{2}} \zeta_{i}
$$

Effect of Torsion on the Seismic Static Torsion Design: The effect of the seismic torsion on the seismic 
static torsion design depends on direct shear, accidental eccentricity and amplified static eccentricity. these last two concepts when used during the design process produce an increment of the direct shear due to torsion for each one of the resistant elements. thus, by defining a torsion amplification factor as taf the total effect due to the seismic torsion can be represented as

$$
T A F_{i}=1+F A E_{i}+F S E_{i}
$$

where $F A E_{i}$ and $F S E_{i}$ represent the amplification factor produced by accidental and amplified static eccentricity, respectively.

By using the factor $T A F$, the static seismic torsion design of the resistant elements can be simplified as is described in the following paragraphs.

Flexible elements. From equations (9), (16) and (18) the effect of the static seismic torsion on the design of the flexible elements is written as:

$$
T A F f_{i}=1+\frac{\zeta_{i}}{\rho^{2}}(\beta+\alpha e)
$$

whereas the total design shear of the flexible elements can be computed as

$$
V_{i}=\operatorname{TAFf}_{i}\left(V_{d i}\right)
$$

Stiff elements. From equations (13), (17) and (16), the $T A F$ for the $i$-th stiff element turns out to be:

$$
T A F s_{i}=1+\frac{\zeta_{i}}{\rho^{2}}(\beta-\delta e)
$$

whereas the total design shear is

$$
V_{i}=T A F s_{i}\left(V_{d i}\right)
$$

Thus, scaling the direct shear value obtained at each one of the structural elements the static seismic torsion design is carried out. On the other hand, the classification of the resistant elements as presented allows a better interpretation of the distribution of the torsion shear among them.

Discussion of The Proposed Equations: As was established, the design of the flexible elements is governed by equation (9); however, the stiff elements design can be governed by equations (12) or (13). from equation (12):

$$
T A F s_{i}=1-\frac{\zeta_{i}}{\rho^{2}}(\beta+\alpha e)
$$

Fig. 3 and Fig. 4 show values of $T A F s_{i}$ computed with equations (23) and (21) for $e=0.3$ and different values of $\zeta_{i}$. It was assumed ${ }^{[10]} \alpha=1.5, \delta=1.0$ and $\beta=0.1$. In general, it can be observed that $T A F s_{i}$ values obtained with equation (23) produce negative and even null values, which means that the computed value of the torsion shear can be greater than or equal to the direct shear. In the first case the resistant elements would be designed for values of the shear forces lower than the direct shear, which represent an unrealistic situation. The extreme case is the second case, in which the value of the design shear that should resist the structural elements could be equal to zero (fig. 3), which means that these elements would not be subjected to lateral forces.

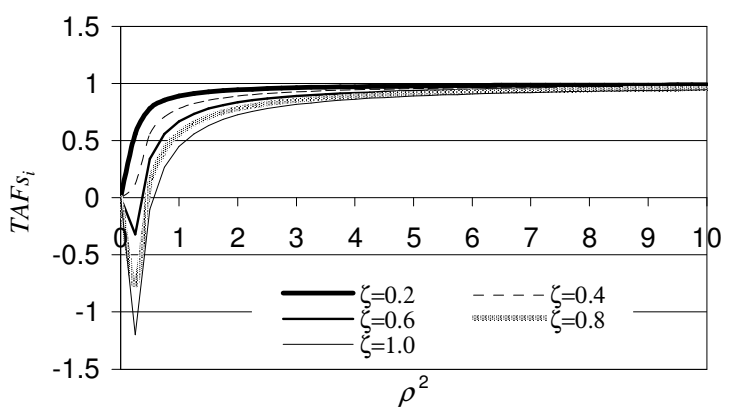

Fig. 3: Torsional Amplification Factor, $T A F s_{\mathrm{i}}$, for $e=0.3$, and different values of $\zeta_{i}$. Computed from equation (23) with $\alpha=1.5$ and $\beta=0.1$ (MFDC$\left.95^{10}\right)$.

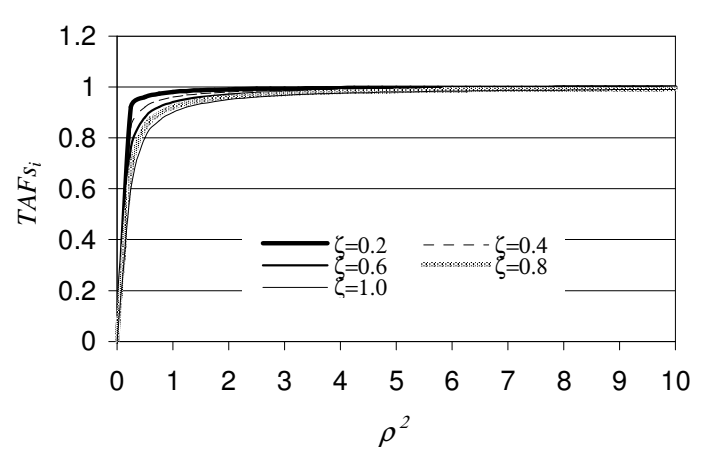

Fig. 4: Torsional Amplification Factor, $T A F s_{i}$, for $e=0.3$, and different values of $\zeta_{i}$. Computed from the equation (21) with $\alpha=1.5$ and $\beta=0.1$ (MFDC-95 $5^{[10]}$ ).

On the other hand, when the $T A F s_{i}$ is computed by using equation (21) negative values are avoided (fig. 4). This confirms that equation (13) will govern the design of the stiff elements. 
It is very important to mention that when the design eccentricity values are used, the location of the $C M$ position could move towards the other side with respect to the $C T$, which means that the denomination of the structural elements also changes. From the point of view of design, that which will be the most "affected" are the stiff elements because, with respect to the $C T$, they will become flexible elements and must be designed as such. In order to define under what circumstances this situation could arise, from equation (21)

$$
\begin{aligned}
& 1 \leq\left[1+\frac{\zeta_{i}}{\rho^{2}}(\beta-\delta e)\right] \\
& \text { and from here } \\
& \delta e \leq \beta
\end{aligned}
$$

Thus, equations (21) and (22) will be applied to the design of the stiff elements only if the value of the normalized structural eccentricity is smaller than or equal to $\beta / \delta$.

On the other hand, from Fig. 4 it can be observed that due to the values of the parameters of equation (21) this does not prevent $T A F s_{i}$ values smaller than unit from developing. In this case total shear values lower than the direct shear will be obtained. Apparently, in the static seismic torsion design practice of the stiff elements in Mexico, this situation is avoided by subtracting only a percentage (not specified) lower than 100 percent of the torsion shear from the direct shear $^{[13]}$.

The above statement was taken into account in the proposal for the new Complementary Technical Norms for Earthquake Design ${ }^{[14]}$, NTC-2001, for the MFDC. These new regulations for seismic torsion design were modified indicating that, "None of the structural elements will have a strength value smaller than what is necessary to resist the direct shear force".

This assumption is based on recent studies ${ }^{[4,15]}$ in which it is shown that structures subjected to intense seismic loads, can exhibit inadequate behaviour when designed by subtracting the torsion shear from the direct shear for the stiff elements.

Based on the above paragraph, and in accordance with the NTC-2001 $1^{[14]}$, equation (21) will be applied to the design of the stiff elements only if the value of the normalized structural eccentricity, $e$, is smaller than or equal to $\beta=0.1$. Otherwise, the total design shear for these elements will be given by the direct shear, which implies that

$T A F s_{i}=1.0$
Regarding the flexible elements, the total design shear calculated with equations (19) and (20) will always produce values greater than the magnitude of the direct shear.

\section{Simplified procedure for torsional shear distribution: Using the concepts developed in the previous paragraphs, the proposed simplified procedure for static seismic torsion design using torsion amplification factors, $P-T A F$, can be used by applying the following steps:}

1. Calculate the storey shear forces, $V_{j}$, from a seismic static analysis.

2. Obtain the direct shear, $V_{d i}$, in the resistant elements.

3. Calculate the coordinates $x_{c t, j}$ and $y_{c t, j}$ of the $C T$ of each one of the storeys.

4. Calculate the coordinates of $C M$ and $C T$ of each one of the storeys and normalize it and calculate the normalized structural eccentricity, $e_{s} / b$. Based on these data, classify the structural elements as flexible or stiff.

5. Compute the radius of gyration $\rho$ for each storey.

6. Using equations (19) and (21) calculate the TAF of the resistant elements.

7. By using the torsion amplification factors calculated in the previous step, correct the direct shear calculated in step 2 with equations (20) and (22). This corrected shear must be sustained by the resistant elements.

Example: An asymmetric three-storey building ${ }^{[16,17]}$ (Fig. 5) is used to illustrate and compare the application of the $P-T A F$ method here described.

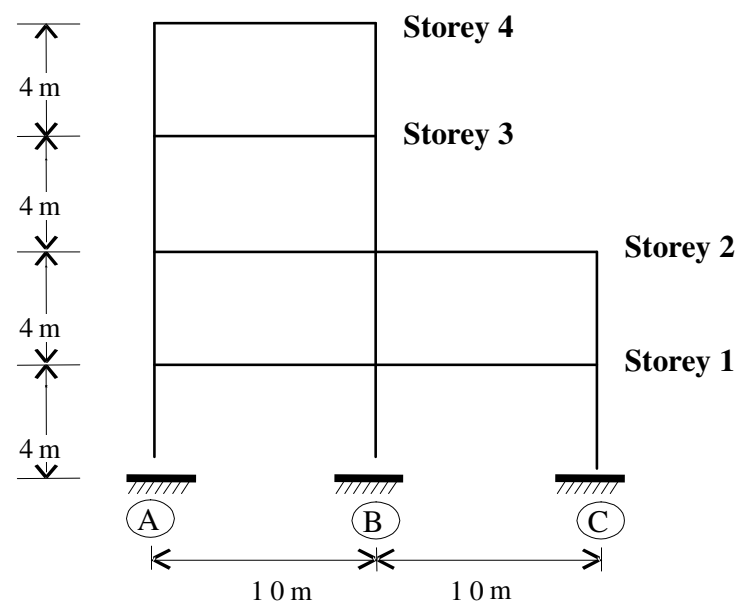

Fig. 5: Studied building ${ }^{[1]}$ 
Am. J. Environ. Sci., 4 (3): 189-197, 2008

Table 1: Lateral forces in the building, direct shear of the resistant elements and calculation of the structural eccentricity ${ }^{[16]}$.

\begin{tabular}{|c|c|c|c|c|c|c|c|c|c|}
\hline \multirow[t]{2}{*}{ Storey } & \multirow[t]{2}{*}{$\begin{array}{c}F_{Y} \\
(\mathrm{KN})\end{array}$} & \multirow[t]{2}{*}{$\begin{array}{c}V_{y} \\
(\mathrm{KN})\end{array}$} & \multicolumn{3}{|c|}{$\begin{array}{l}\text { Direct shear } \\
\quad(\mathrm{KN})\end{array}$} & \multirow[t]{2}{*}{$\begin{array}{l}x_{C T} \\
(\mathrm{~m})\end{array}$} & \multirow[t]{2}{*}{$\begin{array}{l}x_{C M} \\
(\mathrm{~m})\end{array}$} & \multirow[t]{2}{*}{$\begin{array}{c}e_{S}= \\
\left|x_{C M}-x_{C T}\right|\end{array}$} & \multirow[t]{2}{*}{$\begin{array}{l}e \\
(\mathrm{~m})\end{array}$} \\
\hline & & & A & $B$ & $C$ & & & & \\
\hline 4 & 5.54 & 5.54 & 2.22 & 3.32 & --- & 5.99 & 5.0 & 0.99 & 0.099 \\
\hline 3 & 4.15 & 9.69 & 3.82 & 5.88 & ---- & 6.14 & 5.0 & 1.17 & 0.117 \\
\hline 2 & 5.54 & 15.23 & 4.26 & 6.32 & 4.64 & 17.57 & 10.0 & 7.55 & 0.378 \\
\hline 1 & 2.77 & 18.00 & 4.80 & 8.42 & 4.78 & 8.55 & 10.0 & 1.41 & 0.071 \\
\hline
\end{tabular}

Table 2: Computed shear force in each column of resistant element A

\begin{tabular}{clcccccc}
\hline Storey & Type & $\rho^{2}$ & $\zeta_{I}$ & $V_{D}$ & $T A F_{I}$ & \multicolumn{2}{c}{ Total shear $(\mathrm{KN})$} \\
& & & & $(\mathrm{KN})$ & & $P$-TAF & 1.52 \\
Goel-Chopra \\
\hline 4 & flexible & 0.403 & 0.599 & 1.11 & 1.369 & 2.67 & 2.52 \\
3 & flexible & 0.427 & 0.617 & 1.91 & 1.398 & 4.06 & 4.06 \\
2 & flexible & 0.645 & 0.878 & 2.13 & 1.906 & 4.43 & 4.43
\end{tabular}

$*$ The original classification of this element changed because in this storey $e<\beta / \delta$.

Table 3: Total design shear in the resistant elements

\begin{tabular}{|c|c|c|c|c|}
\hline \multirow[t]{2}{*}{ Storey } & \multirow{2}{*}{$\begin{array}{c}\text { Element } \\
\text { Type }\end{array}$} & \multicolumn{3}{|c|}{ Total shear $(\mathrm{KN})$} \\
\hline & & $P-T A F$ & Goel-Chopra & NTC-2001 \\
\hline \multirow{3}{*}{4} & $A$, flex. & 1.52 & 1.52 & 1.52 \\
\hline & $B$, flex.* & 2.07 & & 2.07 \\
\hline & $A$, flex. & 2.67 & 2.67 & 2.67 \\
\hline \multirow[t]{2}{*}{3} & $B$, rig.*** & 2.90 & & 2.94 \\
\hline & $A$, flex. & 4.06 & 4.06 & 4.06 \\
\hline \multirow[t]{3}{*}{2} & $B$, flex. & 4.39 & & 4.39 \\
\hline & $C$, rig. & 2.61 & & 2.61 \\
\hline & $A$, flex.* & 4.43 & 4.43 & 4.43 \\
\hline \multirow[t]{2}{*}{1} & $B$, flex. & 4.79 & & 4.79 \\
\hline & $C$, flex. & 5.07 & & 5.07 \\
\hline
\end{tabular}

\footnotetext{
*The original classification of this element changed because for this storey $e<\beta / \delta$.

**This element does not reduce the design shear by using NTC-2001 ${ }^{[14]}$.
}

Table 4: Over-strength of the resistant elements due to different accidental eccentricity and Torsion Amplification Factors

\begin{tabular}{ccccc}
\hline Storey & Element & $\begin{array}{c}\text { FAEma } \\
e c .(17)\end{array}$ & $\begin{array}{c}\text { TAF } \\
\text { FAEmax }\end{array}$ & $\begin{array}{c}\text { Over- } \\
\text { strength }\end{array}$ \\
\hline 4 & $A$ & 0.248 & 1.467 & 0.07 \\
& $B$ & & 1.396 & 0.02 \\
3 & $A$ & 0.234 & 1.488 & 0.06 \\
& $B$ & & 1.129 & 0.15 \\
2 & $A$ & 0.155 & 1.925 & 0.01 \\
& $B$ & & 1.486 & 0.07 \\
& $C$ & 1.263 & 0.12 \\
& $A$ & 0.962 & 2.392 & 0.30 \\
& $B$ & 2.029 & 0.78 \\
\hline
\end{tabular}


Step 1: The building was designed according to the 1987 México Federal District Code ${ }^{[18]}$. The storey shear forces were calculated from a static seismic analysis considering a system of equivalent forces obtained from a seismic design spectrum. In accordance with Goel and Chopra $^{[16]}$, the lateral forces and direct storey shears are presented in Table 1.

Step 2: If the stiffness of the elements is known, the direct shear on each can be determined by applying equation (2). Alternatively, the direct shear can be obtained by analysing a threedimensional model of the structure restraining the rotation of the slabs around a vertical axis and allowing lateral displacement in only one direction. Using a structural analysis program, the lateral forces are statically applied at the $C M$ of each storey. The resultant shear forces produced in each resistant element will be proportional to their stiffness. In Table 2 the direct shear of the resistant elements computed by Goel and Chopra ${ }^{[16]}$ are presented.

Step 3: Using the direct shear, the $C T$ coordinates of each storey are calculated with the following equation $^{[17]} \quad x_{c t, j}=\Sigma\left(\left(V_{y i, j}-V_{y i, j-1}\right) x_{i}\right) /$ $F_{y j}$ Where $V_{y i, j}$ are the shear forces of the $i$-th resistant element and $F_{y j}$ are the lateral forces, both of them which are on the $j$-th storey. For each floor of the building, in table 1 and in Fig. 6. the location of $C T$ and $C M$ obtained by Goel and Chopra ${ }^{[16]}$ are presented.
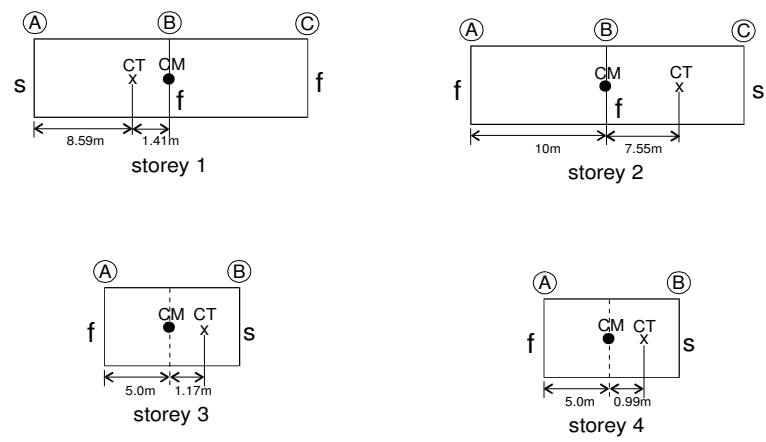

Fig. 6: Storeys plan view of the studied building and classification of the structural elements ( $f=$ flexible, $\mathrm{s}=$ stiff $)$

Step 4: With the coordinates of $C M$ and $C T$ at each storey, the structural eccentricity is calculated. Based on these data, a classification of the resistant elements is carried out. This information is presented in Table 2 for the resistant element $\mathrm{A}$ and for all the resistant elements in Fig. 6

Step 5: If the stiffness of each resistant element is known, the radius of gyration can be computed by using equation (6). If the stiffness values are unknown, the normalized radius of gyration, $\rho_{x j}$ and $\rho_{y j}$, for each of the two orthogonal axes, $x$ and $y$, of the $j$-th storey can be obtained by modifying the equation (6) as follows

$$
\begin{aligned}
& \rho_{x j}=\frac{1}{b_{x j}} \sqrt{\frac{\sum V d y_{i} x_{i}^{2} / d x_{j}+\sum V d x_{i} y_{i}^{2} / d y_{j}}{\sum V d x_{i} / d x_{j}}} ; \\
& \rho_{y j}=\frac{1}{b_{y j}} \sqrt{\frac{\sum V d y_{i} x_{i}^{2} / d x_{j}+\sum V d x_{i} y_{i}^{2} / d y_{j}}{\sum V d y_{i} / d y_{j}}}
\end{aligned}
$$

Where $b_{x j}$ and $b_{y j}$ are the in-plan maximum dimensions of the storey perpendicular to the seismic excitation direction, and $V d x_{i}, V d y_{i}$ and $d x_{j}, d y_{j}$ are the direct shear of the $i$-th resistant element and the corresponding relative displacements of the storey obtained from the structural analyses of step 2 .

In table 2 the computed values of the normalized radius of gyration for each storey are shown.

Step 6: According to the classification of the resistant elements the $T A F$ are computed with equations (19) and (21). Table 2 shows the values of these factors for resistant element A. It is important to mention that on the first storey, because $e<\beta / \delta$, the classification and design of this element changes from stiff to flexible. The reason for this change can be see in Fig 6 . When the design eccentricity is computed, the $C M$ location moves from the right side of the $C T$ towards its left side, and as a consequence, the classification of the stiff element A changes to flexible. Same observation applies for the element B in the four storey.

Step 7: With the torsion amplification factors computed in the previous step, the direct shear values are corrected. The results obtained for the resistant element a are shown in table 2. The last column of this table shows the total shear design values obtained by Goel and Chopra ${ }^{[16]}$. As can be seen, the proposed $P$ $T A F$ produces good agreement. In table 3 the total shear values computed with $P-T A F$ for all the resistant elements and storeys of the building and those obtained by Goel and Chopra $^{[16]}$ with the MFDC-95 ${ }^{[10]}$ are shown. 
Design Approach With The Proposed Ntc-2002 ${ }^{[14]}$ : in the building studied, the $p$-taf by using the proposed ntc- $2002^{[14]}$ will modify the design of the stiff element $b$ in the storey 3 . the total shear values for all the resistant elements and storeys of the building calculated with $p$ taf and corrected with the ntc-2002 $2^{[14]}$ regulations are shown in table 3.some observations about the torsional shear due to accidental eccentricity:for the $j$-th storey of a structure, in an extreme case, the $i$-th resistant element located farthest from the $c t$ will have $\zeta_{i}=1$; thus, equation (15) attains a maximum value of

$$
F A E_{\text {maxj }}=\frac{\beta}{\rho^{2}}
$$

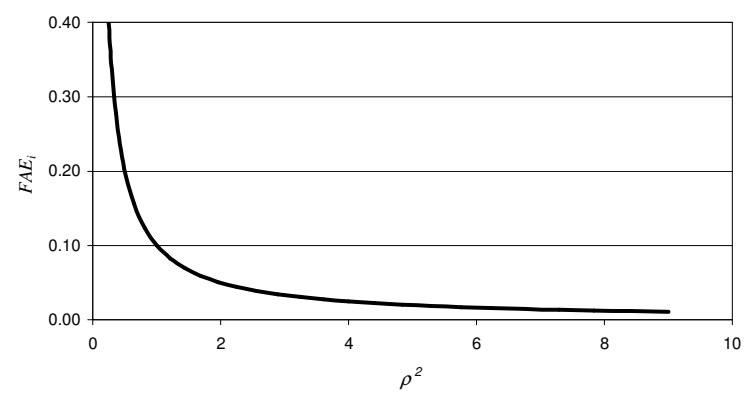

Fig.7: Variation of $\rho^{2}$, and the maximum effect due to the accidental eccentricity; structures designed using the MFDC- $95^{[10]}(\beta=0.1)$.

Fig.7 shows the $F A E_{\max }$ computed with equation (27) for structures designed by using the MFDC- $95^{[10]}$ $(\beta=0.1)$ and for different values of $\rho^{2}$. It can be observed that for values of $\rho^{2}>1$ the value of the $F A E_{\operatorname{maxj}}$ is smaller than 0.1. In this case, the torsion shear due to accidental eccentricity will not exceed 10 percent of the direct shear, whereas for $\rho^{2}=0.5$ the value of $F A E_{\max j}$ is 0.20 .

In general, it can be observed from

Fig.7 that for $\rho^{2}$ values lower than the unit, the accidental eccentricity effect presents a wide range of variations. Thus, the strength increment due to this effect could not be represented by means of only one amplification factor for the whole structure. Nevertheless, it is possible to compute the $F A E_{\max }$ for each storey and to amplify the direct shear values of the resistant elements. Although the implications of this procedure are quite obvious since it saves time when designing the structural elements, however, some of them would be over-designed.
In Table 4 the $F A E$ values for the structural elements of the example calculated with equation (27) are presented. In this table the increment of the resistance obtained when designing the structural elements with these factors is shown. Because the true values of $F A E$ are different for each element, the cost of this over-simplification would cause some of them to be over-designed.

\section{CONCLUSIONS AND RECOMMENDATIONS}

With a view toward simplifying the static seismic torsion design of buildings, in this paper, a new procedure is proposed. In order to attain this goal, it is necessary to review the distribution of shear forces among storey resistant elements in buildings designed by static seismic torsion and to analyse the factors which affect the design eccentricity, particularly accidental eccentricity.

It is demonstrated that the effect of the torsion due to the accidental eccentricity is always additive for the structural elements.

Two other alternatives were explored in order to include the effect of the accidental eccentricity in the static seismic torsion design by means of an amplification factor. In the first alternative an amplification factor for each storey was used. In the second it was found that the value of the accidental eccentricity factor is equal to 10 percent of the direct shear for each of the resistant elements of the storeys where $\rho^{2} \geq 1$. However, as was demonstrated, the real value of this factor is not constant, and for the studied cases the cost of this over-simplification produces an over-design for some of the structural elements. Nevertheless, because for the particular case of the MFDC is not specified an upper limit for the resistance of the structural elements, these alternatives could be optional, although further study is required and is underway ${ }^{20}$.

By means of the P-TAF method, the current procedure for static seismic torsion design is simplified by multiplying the direct shear value of each of the structural elements by its corresponding torsion amplification factor. On the other hand, with the stiff and flexible classification of the resistant elements necessary for the application of the $P-T A F$, it is possible to have a much better interpretation of their shear-force distribution when using the corresponding design equation. 
The results obtained by using the $P-T A F$ proposed here are the same as those calculated by traditional methods.

\section{ACKNOWLEDGMENTS}

To J.N. Dyer ${ }^{\dagger}$, S. Loera and D. Murià-Vila their valuable comments and the critical revision of the written. The initial stage of this study was sponsored by the Dirección General de Obras del Gobierno del Distrito Federal, Mexico.

\section{REFERENCES}

1. Rosenblueth E.,1979. Seismic design requirements in 1976 Mexican Code. Earthquake Engineering and Structural Dynamics, 7:49-61

2. Sadek A.W. and Tso W.K., 1988. Strength eccentricity concept for inelastic analysis of asymmetric structures. 9th World Conference on Earthquake Engineering, V:V-91-V- 96, Tokyo, Japan.

3. Escobar J.A. and Ayala G., 1991. Non-linear seismic response of asymmetric buildings with uncertain parameters. 6th International Conference on Applications of Statistics and Probability in Civil Engineering, CERRAICASP, 6, 1:444-452, Mexico.

4. Escobar J.A., 1994. Respuesta sísmica de estructuras asimétricas inelásticas con propiedades inciertas. Doctoral Thesis, UNAM, Mexico

5. Pekau O.A. and Guimond R., 1988. Accidental torsion in yielding symmetric structures. 9th World Conference on Earthquake Engineering, Tokyo, Japan, V:V-85-V-90

6. De la Llera J.C. and Chopra A.K., 1994. Accidental-torsion in buildings due to stiffness uncertainty. Earthquake Engineering and Structural Dynamics, 23:117-136

7. De la Llera J.C. and Chopra A.K., 1994. Evaluation of code accidental-torsion provisions from building records. Journal of Structural Engineering ASCE, 120:597-616

8. Escobar J.A., 1996. Seismic torsion in non-linear nominally symmetric structures due to random properties. 11th World Conference on Earthquake Engineering, Mexico
9. Escobar J.A. and Ayala G., 1998. Yielding seismic response of code designed single storey asymmetric structures. Earthquake Engineering and Structural Dynamics, 27:525-541

10. MFDC-95, 1995. Normas Técnicas Complementarias para Diseño por Sismo. Gaceta Oficial del Distrito Federal, México

11. Tso W.K. and Wong C.M., 1993. An evaluation of New the Zealand Code torsional provision. Bulletin of New the Zealand National Society for Earthquake Engineering, 26, 2:194-207

12. Paulay T., 1997. Are existing seismic provisions achieving the design aims?. Earthquake Spectra, May, 13, 2:259-279

13. Damy J., 1988. Comentarios al inciso 8.6 de las Normas Técnicas Complementarias para Diseño por Sismo. Ingeniería Sísmica, 33:66-99

14. NTC-2002, 2002. Propuesta de las Normas Técnicas Complementarias en Materia de Diseño estructural. Centro Nacional de Prevención de Desastres, Mexico

15. Escobar J.A., Gómez R. and Páez A., 1999. Evaluación de criterios de diseño por torsión. Internal report for the Dirección General de Obras del Gobierno del Distrito Federal, Instituto de Ingeniería, UNAM, Mexico

16. Goel R.K. and Chopra A.K., 1993. Seismic Code analysis of building without locating centres of rigidity. Journal of Structural Engineering ASCE, 119:3039-3055

17. Tso W.K., 1990. Static eccentricity concept for torsional moment estimations. Journal of Structural Engineering ASCE, 120:597-616

18. Gómez R. and García-Ranz F., 1988. The Mexico earthquake of September 19, 1985complementary norms for earthquake resistant design. Earthquake Spectra, 4, 3:441-460 